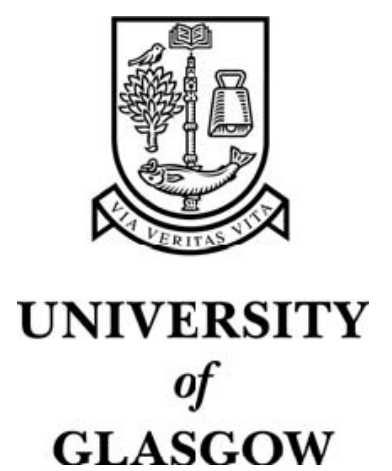

Johnson, N.P. and Khokhar, A.Z. and Chong, H.M. and De La Rue, R.M. and Antosiewicz, T.J. and McMeekin, S. (2006) A review of size and geometrical factors influencing resonant frequencies in metamaterials. Opto-Electronics Review 14(3):pp. 187-191.

http://eprints.gla.ac.uk/3715/ 


\title{
A Review of Size and Geometrical Factors Influencing Resonant Frequencies in Metamaterials
}

\author{
Nigel P Johnson*1, Ali Z Khokhar ${ }^{1}$, Harold M Chong ${ }^{1}$, Richard M De La Rue ${ }^{1}$, Tomasz J Antosiewicz ${ }^{1,2}$ \\ and Scott McMeekin ${ }^{3}$ \\ ${ }^{1}$ Department of Electronics and Electrical Engineering, University of Glasgow, Glasgow G12 8LT, UK \\ ${ }^{2}$ Permanent address: Faculty of Physics, Warsaw University, 02-093 Warsaw, Poland \\ ${ }^{3}$ Department of Engineering Science and Design, Glasgow Caledonian University, Glasgow G4 0BA, UK
}

\begin{abstract}
Although Metamaterials and so-called left-handed media have originated from theoretical considerations - it is only by their practical fabrication and the measurement of their properties that they have gained credibility and can fulfil the potential of their predicted properties. In this review we consider some of the more generally applicable fabrication methods and changes in geometry as they have progressed, exhibiting resonant frequencies ranging from radio waves to the visible optical region.
\end{abstract}

Keywords: Metamaterials, Fabrication, Lithography, Geometry

\section{Introduction}

Metamaterials or left-handed media were first introduced by Veselago [1] - and rediscovered with proposals for implementation by Pendry [2]. However, the first practical demonstration of one of the key predicted properties, negative refraction, was made by Smith [3] at microwave frequencies. This relatively large-scale experiment, with a unit cell of approximately $1 \mathrm{~cm}$, mirrors the development of photonic crystals (PhCs). The first threedimensionally periodic structure to exhibit a full photonic band gap did so at microwave frequencies and was produced by drilling macroscopic holes in a proprietary dielectric material. This process produced a Yablonovite structure, named after the co-predictor of its properties [4]. In both cases the inevitable move to shorter wavelengths, firstly to the communications wavelength range around 1.5 microns - and subsequently to the visible part of the spectrum, have required a variety of fabrication techniques such as optical or e-beam lithography. It is worth mentioning at this stage that $\mathrm{PhCs}$ can exhibit negative refraction due to the curvature of their band-structure and their strongly anisotropic nature [5]. PhCs have been included within the definition of metamaterials - as materials that are not found in nature. But, in fact, Nature has produced a variety of $\mathrm{PhCs}$, including opal and the wings of the morpho butterfly. The negative refraction exhibited by $\mathrm{PhCs}$ is not due to the fact that the structure possesses negative $\mu$ and

* e-mail: njohnson@elec.gla.ac.uk

tel. $+44(0) 1413304110$ negative $\varepsilon$ - terms that are arguably inappropriate [6] for situations where the wavelength is on the order of the lattice constant of the $\mathrm{PhC}$.

Within the definition of metamaterials it is also perverse to exclude all materials that do not simultaneously exhibit negative $\mu$ and negative $\varepsilon$, i.e. so-called double negative (DNG) materials. Such a definition would exclude the use of a thin silver film for the creation of a super-lens, as well as devices based on controlling the resonances exhibited by subwavelength structures. So we are left with a loose definition of metamaterials, over the whole electromagnetic spectrum, as being sub-wavelength structures that use constituent materials that are not necessarily metallic and are not necessarily periodic. In short, a variety of structures - and this may help in explaining the rapid rise in the popularity of this topic.

\section{Microwave and Longer Wavelength Techniques}

The first major success in demonstrating DNG materials was through the use of printed circuit board technology. This is a well established technology, in which copper-coated sheet is patterned by use of UV positive photoresist and then etched in a mixture of ferric chloride and hydrochloric acid. Double-sided boards can also readily facilitate combinations of, for instance, split ring resonators (SRRs) and wires. Indeed the combination of wires and double SRRs was considered essential to produce the observed simultaneous double negative properties [3]. One of the main advantages of working at this scale $a=8 \mathrm{~mm}$ 
$(\lambda \sim 60 \mathrm{~mm})$ is that three-dimensional structures can be slotted together from the constituent materials. The practical size limit is determined by the metal strip width and the thickness of the boards which limits the stacking density of the circuit boards.

Even larger structures in the form of spiral-wound, insulated metallic sheets [7] have been successfully used to transfer the magnetic image of an antenna. Many of the so called Swiss-roll structures are stacked together to form an array area of approximately $10 \mathrm{~cm} \times 10 \mathrm{~cm}$, with a lattice constant, $a$, of approximately $1 \mathrm{~cm}$. At this scale, the individual components of the metamaterial are much smaller than the wavelength of $15 \mathrm{~m}$ that corresponds to the resonant frequency of $20 \mathrm{MHz}$, giving $\lambda / \mathrm{a} \sim 1500$.

\section{Near-IR and Optical Techniques}

The main driver for increasing the resonant electric and magnetic frequencies of metamaterials is reduction in size, together with some key changes in geometry. Optical and e-beam lithography are well suited to the fabrication of planar devices: however multilayer structures are much more challenging. Planar SRR structures have $\lambda / \mathbf{a} \sim 10$ - much less than the Swiss roll structures.

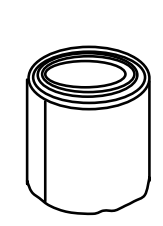

(a)

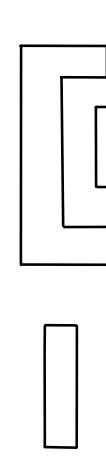

(e)

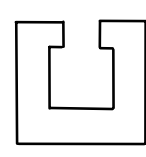

(c)

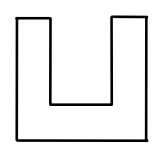

(d)

(b)

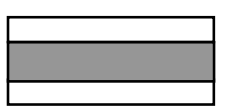

(f)

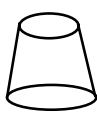

(g)
Fig. 1. Schematic representation of various structures leading to resonances at wavelengths ranging from $15 \mathrm{~m}$ to the visible: (a) Swiss roll, (b) double SRR with cut wire, (c) single SRR, (d) 'U' shaped SRR, (e) in-plane double cut wires, (f) stacked double cut wires, $(\mathrm{g})$ pairs of cones.

In moving from double ring structures to single ring SRRs the resonance is shifted to shorter wavelengths but becomes weaker, since $\lambda / \mathrm{a}$ is further reduced due to the smaller capacitance of the single ring SRR.
Several alternative geometries are shown schematically in Fig. 1. One of the first examples of significant shrinkage and simplification of structures was the work by Linden et al [8]. The plasmon resonances and the LC resonance excited by the electric field were considered, because the LC resonance is inaccessible to the magnetic field for perpendicular excitation, in either TE or TM polarisation. Using arrays of the single SRR on ITO coated glass; the polarisation-sensitive LC resonance was demonstrated at $3 \mu \mathrm{m}$ wavelength. Our own group has produced similar structures fabricated on silicon [9]. A rationale for using silicon is that it lends itself to integration with other technologies, either optical or electro-optical, and could eventually lead to metamaterials with enhanced functionality. In Fig. 2 we show the disappearance of the electricallyinduced magnetic resonance, as the rings are closed. By electrically or optically inducing electron-hole pairs in the silicon substrate a switching action can be achieved. This effect has been demonstrated recently in low temperature grown GaAs [10].

\section{Material Effects - substrate}

One disadvantage of using a high dielectric constant substrate like $\mathrm{Si}$ or $\mathrm{GaAs}$, in comparison with a lower dielectric constant material such as silica, is that both the plasma frequency and the LC resonance are shifted to longer wavelengths. At around $1.5 \mu \mathrm{m}$, in the specific structures that we have measured, the shift is $\sim 20 \%$ (see Fig. 3). The shift, with air above the substrate, is considerably less than would be expected if the metallic patterns were fully embedded in the respective dielectric media alone.

The positions of the plasmon resonance and the LC resonances can be modelled with commercial FTDT software [11]. The agreement is very good for larger structures where rounded corners make up only a small part of the structure. It is less so where small structure geometries, with more rounded corners, are approximated by rectangles (Fig. 2).

\section{Equivalent Circuit Model}

Insight into factors affecting the positions of the resonances can also be obtained by the use of equivalent circuit models. The LC electrical resonance of a metal SRR is given by $\omega_{L C}=\left(L_{T} C_{T}\right)^{-1 / 2}$ where $L_{T}$ is the total inductance formed by the combination of the self inductance of 

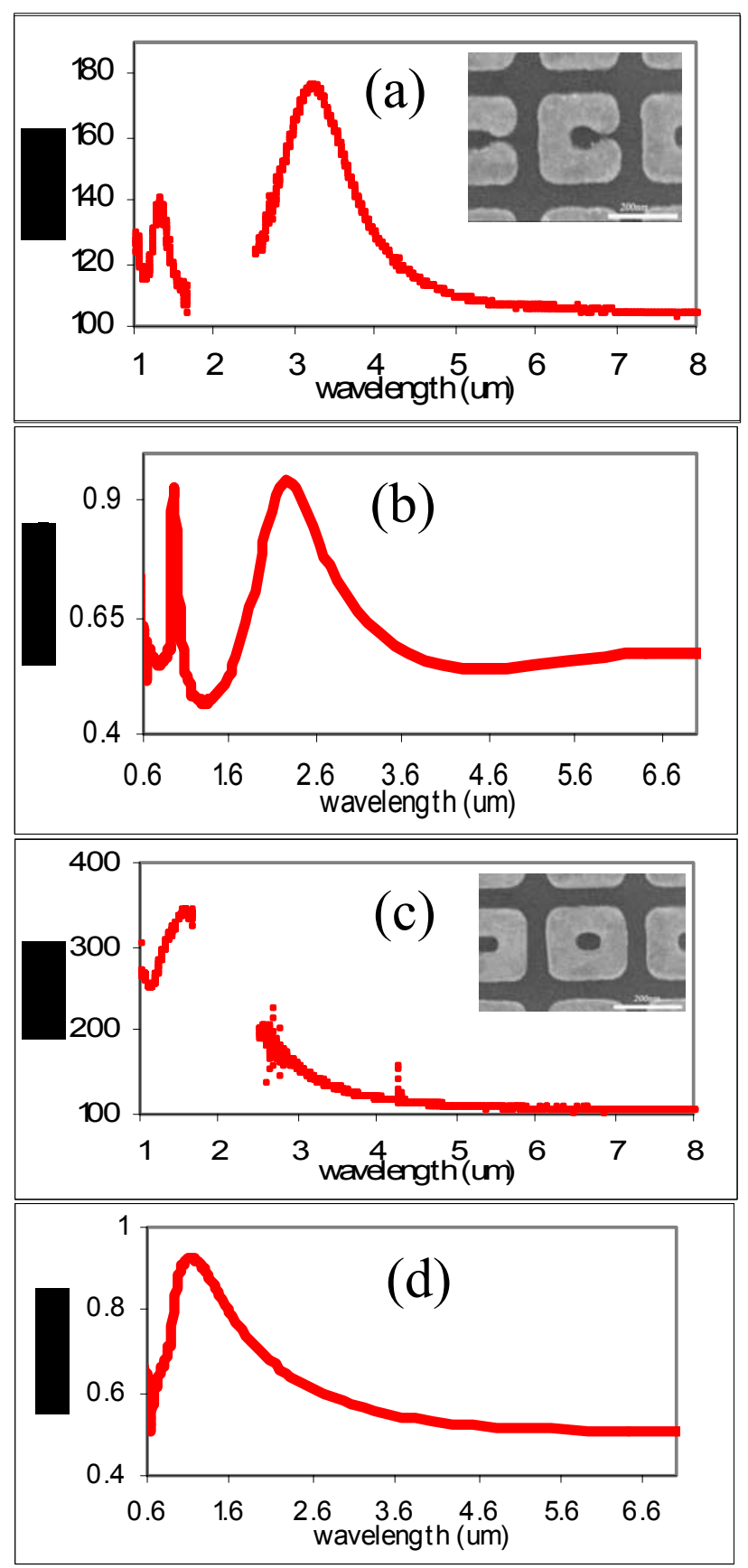

Fig. 2 (a) Experimental reflectance spectra of open rings, (b) simulation; (c) experimental closed, (d) simulation. TE polarisation is vertical.

the rectangular loop forming the SRR element, $L_{\text {Ring, }}$, and the mutual inductance formed between the conducting arms of neighbouring SRR elements, $L_{m u t}$. However, in order to calculate the total capacitance of the SRR element, $C_{T}$, it is necessary to take into account both the parallel plate and co-planar capacitances formed between the individual SRR elements, $C_{e}$, and the capacitance formed by the gap in the SRR structure, $C_{g}$. The parallel plate capacitance of both components can be calculated from the standard equation $\mathrm{C}=\varepsilon_{0} \quad \varepsilon_{\mathrm{r}}$ (area/separation), while the co-planar capacitance can be calculated by using conformal mapping techniques that take into account the field that is present in the substrate. The general form of the equation for the co-planar capacitance per unit length for two metal strips of width $p$ and separated by distance $\mathrm{q}$, is given by [12]:

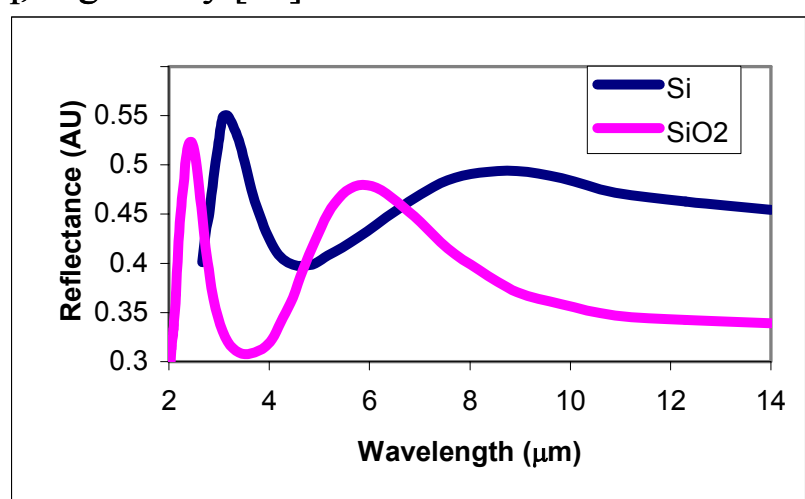

Fig. 3 FTDT simulations of identical gold SRRs on silicon and silica substrates $l=564, a=750, w=130$, $g=120 \mathrm{~nm}, t=30 \mathrm{~nm}$

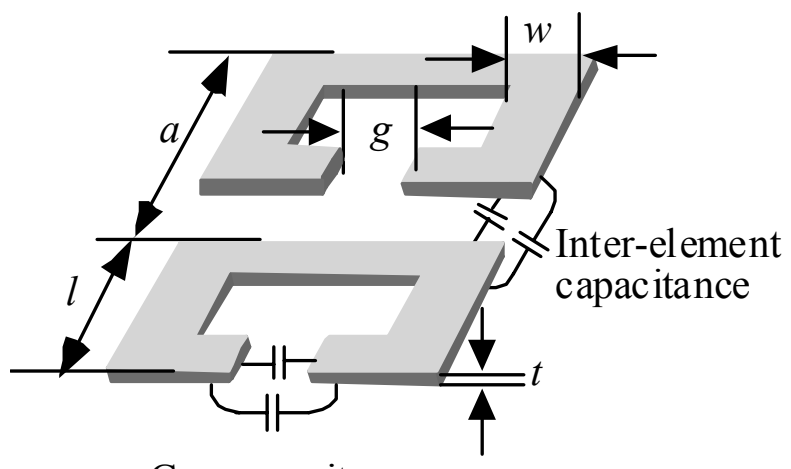

Gap capacitance

Fig. 4 Equivalent circuit model taking into account the co-planer capacitance.

$$
C_{c p}=\frac{\left(\varepsilon_{r}+1\right) \varepsilon_{0}}{2} \frac{1}{\pi} \ln \left[2 \frac{1+\sqrt{k^{\prime}}}{1-\sqrt{k^{\prime}}}\right] \quad F / m
$$

where $k^{\prime}$ is a geometrical factor given by:

$$
k^{\prime}=\sqrt{\left(1-\left(\frac{p}{p+2 q}\right)^{2}\right)}
$$


The shorter wavelength resonances shown in Fig. 2 and Fig. 3 are the reduced plasma frequencies of the metal [13] and are a collective property of the SRR array. This resonance has relatively low dependence on shape and, at frequencies in much of the infra-red part of the spectrum, is also not strongly dependent on the particular metal used. In the simplest model, the resonant frequency of the peak is dependent upon the dilution of the metal and is inversely proportional to the natural logarithm of the filling fraction given by the ratio of the period of the SRR array to the radius of extended cylindrical pillars [13]. To take into account the more complex geometry of the SRR elements - and the fact that they are closely packed a factor $\mathrm{F}$ is introduced, which is the area of exposed silicon in a unit cell, i.e. the total area of the unit cell minus the area covered by the metallic SRR.

A modified expression for the reduced plasma frequency may then be written as:

$$
\omega_{p}^{2}=\frac{c^{2} \cdot 2 \pi}{\left.a^{2} \cdot \ln \left(a^{2} / F\right)\right) \cdot n_{\text {eff }}^{2}} \operatorname{rads}^{-1}
$$

where $n_{\text {eff }}$ is the effective refractive index of the dielectric medium at the frequency of the infra-red radiation interacting with the SRR array. The value of $n_{\text {eff }}$ is approximately $70 \%$ of the value of silicon and is related to the distribution between silicon and air of the electric flux density interacting with the SRR array. As the metal area of an individual SRR increases, for a fixed unit cell area, F decreases - and the plasma frequency also decreases. This effect is greatest at the smallest spacing between SRRs, where the position of the peak diverges substantially from the simple theory. A more detailed account is given elsewhere [14].

\section{Material Effects - metal}

The choice of metal to use in making metamaterial structures is usually between gold $(\mathrm{Au})$, silver $(\mathrm{Ag})$, copper $(\mathrm{Cu})$ and aluminium (Al). Copper is clearly suited for circuit boards when the patterns are formed by subtractive etching but is not so easily deposited for use in sub-micrometre structures fabricated by lift-off. Fitting the Drude model to experimental measurements $[15,16]$ shows that aluminium has significantly smaller $\operatorname{Re}(\varepsilon)$ and significantly larger $\operatorname{Im}(\varepsilon)$ in the range down to a wavelength of $0.5 \mu \mathrm{m}$ compared to the other metals considered. However simulations on cubes of metal with a side-length of $250 \mathrm{~nm}$ do not show significant absorption until wavelengths below $1.5 \mu \mathrm{m}$. The choice of metal could also be affected by eventual larger volume production. Gold is traditionally avoided in a silicon VLSI foundry because of its ability to form deep levels in the silicon. Aluminium is the most commonly chosen metal in silicon VLSI - and has a higher bulk plasma frequency than silver or gold. In our own measurements on SRRs formed from $\mathrm{Al}, \mathrm{Au}$ and Ag no significant difference could be attributed to the metal at wavelengths down to $2.5 \mu \mathrm{m}$ [14]. SRRs formed from silver are noticeably granular and prone to diffusion (see Fig. 5)

A progression of structures that gradually open up the SRR elements - firstly into a ' $U$ ' shape and then a pair of parallel wires [17] - has shown a reduction in the wavelength for the magnetic resonance.

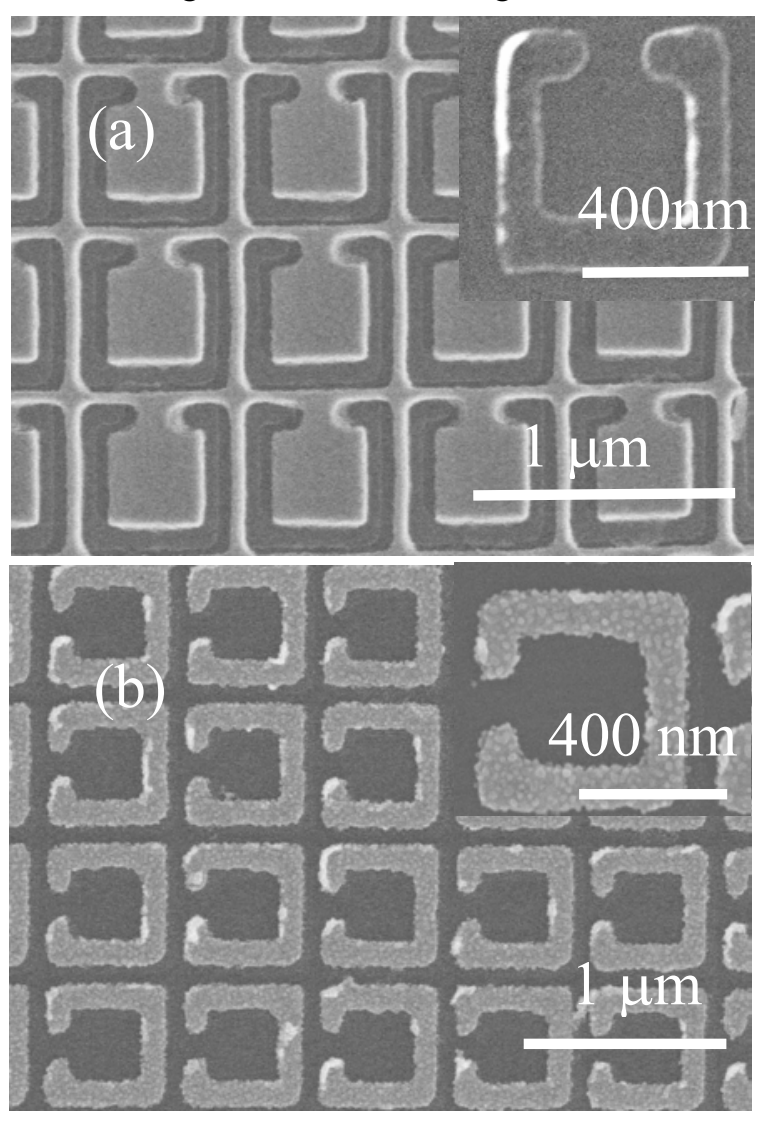

Fig. 5 (a) Aluminium and (b) silver SRRs - note the granularity of the silver metal.

The progression lowers the capacitance of the ring structures, while opening the bottom of the ring results in two capacitors in series - and further shifts the resonance towards the blue.

The disadvantage of periodic array structures with pairs of cut wires is that the ratio of wavelength to 
lattice constant is reduced from $\lambda / \mathrm{a} \sim 10$ for a SRR to $\lambda / \mathrm{a} \sim 2$ for cut wires - effectively moving towards the region of $\mathrm{PhC}$ behaviour. A double-wire system, in which the wires are formed on top of each other and are separated by a dielectric layer, has the distinct advantage that, when excited with k-vector perpendicular to the plane of the double wires, the LC resonance can be excited by both the electric and magnetic fields. However careful tuning of the parameters for the pairs of cut wires is necessary to obtain demonstration of negative refractive index at $1.5 \mu \mathrm{m}[18]$. An example of silica sandwiched by double gold wires, fabricated on an ITO-covered glass substrate is shown in Fig. 6. Structures based on such pairs of wires combined with long wires to drive $\varepsilon$ negative have been demonstrated at $14 \mathrm{GHz}$ and have been proposed for the $\mathrm{THz}$ range [19].

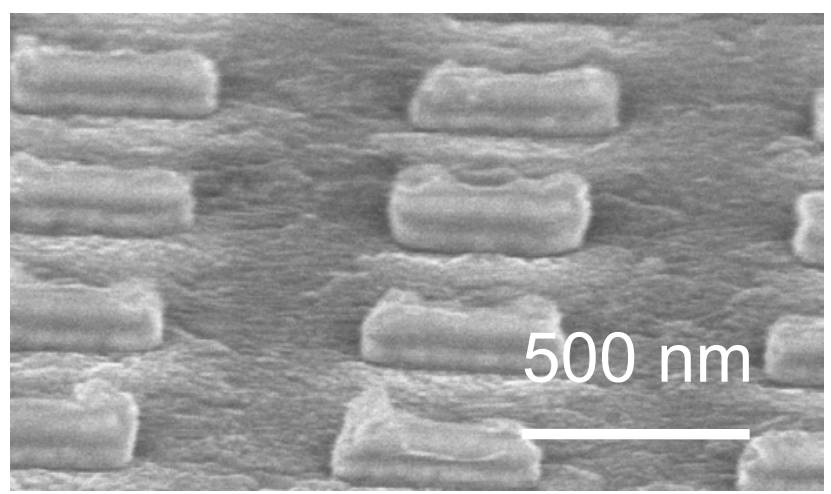

Fig. 6 Example of several stacked double cut wires formed from $50 \mathrm{~nm}$ silica sandwiched between two gold layers $\sim 30 \mathrm{~nm}$.

The last geometry considered in this short review is the pair of cones. By simplifying the geometry Grigorenko et al [20] have demonstrated negative permeability at visible wavelengths. The cone structures are deliberately shaped to reduce the number of coupled modes. As the structures are relatively simple they can be made small $(\sim 100 \mathrm{~nm})$ and act like double open ended SRRs. Using normal incidence light (ie k-vector into the top of the cones) TE and TM polarisations can easily be probed. The anti-symmetric plasmon resonance corresponding to the magnetically excited resonance is at $550 \mathrm{~nm}$ (the green) and the lattice spacing between pairs of cones is for the strongest resonance is $400 \mathrm{~nm}$ give a very small $\lambda / a \sim 1.4$. This is reflected in the weak nature of the resonance which was enhanced using a thin dielectric coating of glycerine.

\section{Conclusions}

The more complex a structure, for example the more turns and more surface area per unit volume a structure possesses, the more inductance and capacitance per unit volume. As L and $\mathrm{C}$ are reduced, the resonant frequencies reduce - but also the values of $\lambda / \mathrm{a}$ decrease, resulting in a reduction in the sharpness and intensity of the $\mathrm{LC}$ resonance.

With knowledge and understanding gained from various fabrication methods and materials, backed by theory and simulation, it will surely only be a short time before double negative behaviour will be pushed further into the visible region.

\section{Acknowledgements}

We acknowledge the support of the EU Network of Excellence, Metamorphose.

\section{References}

1. V.G. Veselago, "Electrodynamics of substrates with simultaneously negative electrical and magnetic permeabilities" Sov. Phys. Usp. 10, 5-13, (1968)

2. J.B. Pendry, "Negative refraction makes a perfect lens", Phys Rev Lett, 85, 3966-3969 (2000).

3. D.R. Smith, W.J. Padilla, D.C. Vier, S.C. NematNasser, and S. Schultz, "A Composite Media with Simultaneously Negative Permeability and Permittivity”, Phys. Rev Lett. 84, 4184-4187, (2000)

4. E. Yablonovitch, Phys Rev Lett 58, 2059 (1987)

5. M. Notomi, "Theory of light propagation in strongly modulated photonic crystals : Refraction like behaviour in the vicinity of the photonic band gap," Phys. Rev. Lett. B62, 10696-10705 (2000)

6. D. R. Smith, J. B. Pendry and M. C. K. Wiltshire, Science 305, 788 (2004)

7. M. Wiltshire, J. Hajnal, J. Pendry, D. Edwards, and C. Stevens "Metamaterial endoscope for magnetic field transfer: near field imaging with magnetic wires", Optics Express, 11, 709 (2003)

8. S. Lindon, C. Enkrich, M. Wegener, J Zhou, T. Koschny and C. M. Soukoulis, Science 306, 1351 (2004)

9. N. P. Johnson, A. Z. Khokhar, H. M. Chong, C. Jin, J. Mandel, S. McMeekin and R M De La Rue, Increasing Optical Metamaterials Functionality Proc. SPIE 5955 595500-1-59550O-6 (2005).Metamaterials Ed Tomasz Szoplik, Ekmel Ozbay, Costas M Soukoulis, Nikolay I Zheludev

10. W. J. Padilla A. J. Taylor C. Highstrete M. Lee and R. D. Averitt, Dynamical Electric and Magnetic Metamaterial Response at Terahertz Frequencies Phys. Rev. Lett. 96, 107401 (2006) 
11. RSoft Design Group 400 Executive Blvd. Suite 100 Ossining, NY 10562

12. H. A. Wheeler, IEEE Trans Microwave Theory Tech. MTT-13, 172-185 (1965).

13. J. B. Pendry, A. J. Holden, D. J. Robbins and W. J. Stewart, J. Phys.: Condens. Matter 10, 4785- 4809 (1998)

14. N. P. Johnson, A. Z. Khokhar, H. M. H. Chong, R. M. De La Rue and S. McMeekin, Characterisation at infrared wavelengths of metamaterials formed by thin-film metallic split-ring resonator arrays on silicon, Electronic Letters. Accepted for publication.

15. I. El-Kady, M. M. Sigalas, R. Biswas, K. Ho and C. M. Soukoulis, Phys Rev B 62, 15299 (2000)

16. M. A. Ordal, L. L. Long, R. J. Bell, S. E. Bell, R. R. Bell, R. W. Alexander,Jr. and C. A. Ward, Appl. Opt 221099 (1983); M. A. Ordal, R. J. Bell, R. W. Alexander,Jr. ,L. L. Long and M. R. Querry, ibid 2444 93 (1983)

17. G. Dolling, C. Enkrich, M. Wegener, J. F. Zhou C. M. Soukoulis and S. Linden, Opts Letts 30, 3198 (2005)

18. V. M. Shaleav, W. Cai, U. K. Chettiar, H-K. Yuan, A. K. Sarychev, V. P. Drachev and A. V. Kildishev, Negative index of refraction in optical metamaterials, Optics letters 30, 3356 (2005)

19. J. Zhou, L. Zhang, G. Tuttle, T. Koschny and C. M. Soukoulis, Phys Rev B 73, 041101 (2006)

20. A. N. Grigorenko, A. K. Geim, H. F. Gleeson, Y. Zhang, A. A. Firsov, I. Y. Khrushchev and J. Petrovic, Nature 438, 335 (2005) 
